\title{
Optimization of quantum graphs using heuristic algorithms
}

\author{
Ichio Kikuchi ${ }^{1}$, Akihito Kikuchi $^{2} *$ \\ ${ }^{1}$ Internationales Forschungszentrum für Quantentechnik \\ ${ }^{2}$ International Research Center for Quantum Engineering, Tokyo
}

March 5, 2021

\begin{abstract}
This essay explains the optimization (or the designing) of quantum graphs through heuristic algorithms.
\end{abstract}

\section{Introduction}

Quantum graph [1, 2] is an item composed of B edges with length $L_{i}(i=1, \ldots B)$ and $\mathrm{V}$ vertexes upon which quantum mechanics is defined by the following functional.

$$
F:=\sum_{b=1}^{B} \int_{0}^{L_{b}} d x_{b}\left(\left|\frac{1}{i} \frac{d \psi_{b}}{d x}+A_{b} \psi_{b}\left(x_{b}\right)\right|^{2}+w_{b}\left(x_{b}\right)\left|\phi_{b}\left(x_{b}\right)\right|^{2}\right)+\sum_{j=1}^{V} \lambda_{j}\left|\phi_{j}\right|^{2}(1)
$$

Here $\psi_{b}\left(x_{b}\right)$ is the wave function defined at the edge $\mathrm{b}$ with the local coordinate $x_{b}$ such that $0<=x_{b}<=L_{b} ; \phi_{j}$ is the wavefunction at the vertex $j ; A_{b}$ is the vector potential and $w_{b}(x)$ is the potential function on the edge; $\lambda_{j}$ is the variables which determines the continuity condition on the vertices.

Without the edge potential, the wave function in the edge $b$ between two vertex $i$ and $j$ is explicitly written as

$$
\phi_{b}(x ; k)=\frac{\exp \left(-i A_{b} x\right)}{\sin \left(k L_{b}\right)}\left(\phi_{j} \exp \left(i A_{b} L_{b}\right) \sin \left(k L_{b}\right)-\phi_{i} \sin \left(k\left(x_{b}-L_{B}\right)\right)\right) .
$$

*akihito_kikuchi@gakushikai.jp (The corresponding author) 
To determine the wavenumber $k$, we require that the variation of the energy functional $\delta F$ equals zero. Then we obtain the following relation upon the vertex.

$$
\sum_{b} f_{b}\left(\frac{d}{d x_{b}}+i A_{b}\right) \psi_{b}\left(x_{b}^{i}\right)=\lambda_{i} \phi_{i}
$$

where the summation for $b$ is taken over the whole of the edges connected to the vertex $i$. Here we use the weight $f_{i}$ upon the coordinates of the vertex $x_{b}^{i}$ :

$$
f_{i}= \begin{cases}-1 & x_{b}=L_{b} \\ 1 & x_{b}=0\end{cases}
$$

In the case of zero edge potential, the secular equation is explicitly given by functions in the following:

$$
h_{i i}(k)=\frac{\lambda_{i}}{k}+\sum_{b} \cot \left(k L_{b}\right),
$$

and

$$
h_{j i}(k)=-\frac{\exp \left(i \omega_{b} A_{b} L_{b}\right)}{\sin \left(k L_{b}\right)}
$$

In the definition of $h_{j i}$, the function is non-zero only between the nodes connected by an edge. The weight $\omega_{b}$ is 1 or -1 ,according to the choice of the head and the tail of the edge between nodes $i$ and $j$. The wavefunction on the vertices are computed (up to the constant) by the linear equation:

$$
\sum_{j} h_{j i} \phi_{j}=0 \text { for } \mathrm{i}=1, \ldots, \mathrm{V}
$$

For this reason, the energy spectrum $k^{2}$ is the zeros of the determinant $\operatorname{det}(\mathrm{h})=0$; once the zeros of $\operatorname{det}(\mathrm{h})$ is determined, the $\phi_{i}$ are given by the nonzero solution of the linear equation; we also determine the wavefunctions in the edges.

As one can see without difficulty, one can design a quantum graph so that it shows some desired property. To this end, one has to deal with those equations, and one has to adjust various parameters with the constraint of $\operatorname{det} \mathrm{h}(\mathrm{k})=0$. In the following section, we try to demonstrate several model problems of the optimization of quantum graphs using heuristic algorithms. 


\section{Optimization on quantum graphs}

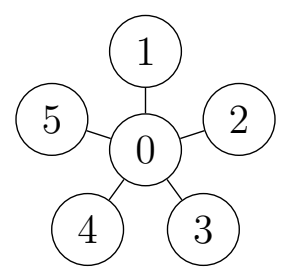

Figure 1: A star-like graph. We allot the index 0 at the center node and indices 1 ...B to the peripheral ones in the latter computations.

For computations, let us use star-like graphs as in Fig. 1 (with $\mathrm{B}=5$ ), in which the edges are emitted outward from one central node to the peripheral ones, while no pair of the latter are connected. We assume no edge and vector potentials $\left(w_{j}=0, A_{j}=0\right.$ for $\left.j=1, . . \mathrm{B}\right)$. In this case, the energy spectrum is determined by the equation

$$
0=\operatorname{det} h\left(k_{0}\right)
$$

where the matrix entries are given by

$$
\begin{gathered}
h_{00}(k)=\frac{\lambda_{0}}{k}+\sum_{i=1}^{B} \cot \left(k L_{i}\right), \\
h_{j j}(k)=\frac{\lambda_{j}}{k}+\cot \left(k L_{j}\right),
\end{gathered}
$$

and

$$
h_{0 j}(k)=-\frac{1}{\sin \left(k L_{j}\right)} .
$$

The energy spectrum is simply given by $k_{0}^{2}$.

At first, let us try to obtain the zeros of the determinant defined above. We use the particle swarm optimization [3]. The algorithm is summarized as follows. Let $\left\{\mathbf{s}_{j}(t)\right\}$ and $\left\{\mathbf{v}_{j}(t)\right\}$ be the coordinates and the velocities of sampling particles $(j=1, \ldots \mathrm{n})$ at the time $t$, at which the objective function is computed. Each particle keeps the memory of the past best-position $\left\{\mathbf{p}_{j}\right\}$, at which the objective function has taken the best value. Likewise, the entire swarm keeps the past best-position $\mathrm{g}$, at which some of the participants have achieved the best value of the objective function $f(\mathbf{x})$.

1 Initialization. Start from the set of particles, for which the velocity and the coordinate of each particle are chosen randomly. 
2 Evaluation of objective function. For each particle, compute the objective function $f\left(\mathbf{s}_{j}\right)$ at the coordinate $\mathbf{s}_{j}(t)$. The best position $\mathbf{s}_{b e s t}$ in the present group (concerning the evaluated objective function) is found now. Then the past best-positions of each particle $\left\{\mathbf{s}_{j}(t)\right\}$ and that of the entire group $(\mathbf{g})$ are updated as follows. For each particle, if $f\left(\mathbf{p}_{\mathbf{j}}\right)>f\left(\mathbf{s}_{\mathbf{j}}(t)\right)$, then replace $\mathbf{p}_{j}$ with $\mathbf{s}_{j}(t)$.; else keep $\mathbf{p}_{j}$. For the group, if $f(\mathbf{g})>f\left(\mathbf{s}_{\text {best }}\right)$, then replace $\mathbf{g}$ with $\mathbf{s}_{\text {best }}$; else keep $\mathbf{g}$.

3 Time evolution. The velocity and the coordinate are updated by the following formula.

$$
\begin{gathered}
\mathbf{v}_{j}(t+1)=W \cdot \mathbf{v}_{j}(t)+C_{1} \cdot r_{1} \cdot\left(\mathbf{p}_{j}-\mathbf{s}_{j}(t)\right)+C_{2} \cdot r_{2} \cdot\left(\mathbf{g}-\mathbf{s}_{j}(t)\right) \\
\mathbf{s}_{j}(t)=\mathbf{s}_{j}(t)+\chi \cdot \mathbf{v}_{j}(t)
\end{gathered}
$$

The parameter $W$ is the inertia which leads the group to a standstill in the end; $C_{1}, C_{2}$, and $\chi$ are the parameter which determines the extent of the jump of the velocities and the positions from one step to the next; the alternation of the velocity and the positions are also regulated by random numbers $r_{1}$ and $r_{2}$.

4 Go back to 2 .

In this work, we judge the convergence by the condition of whether the sampling particles gather at the local minimum of the objective function. We use the variation of the particle coordinates as the criterion.

\subsection{Computation of energy spectrum}

As a quantum system has several numbers (indeed infinite numbers) of energy spectra, we have to investigate the problem with the assumption that several local minima would coexist. The $k$-dependence of the determinant in our model is shown in Fig.2. We see two zeros of the determinant in this figure. 


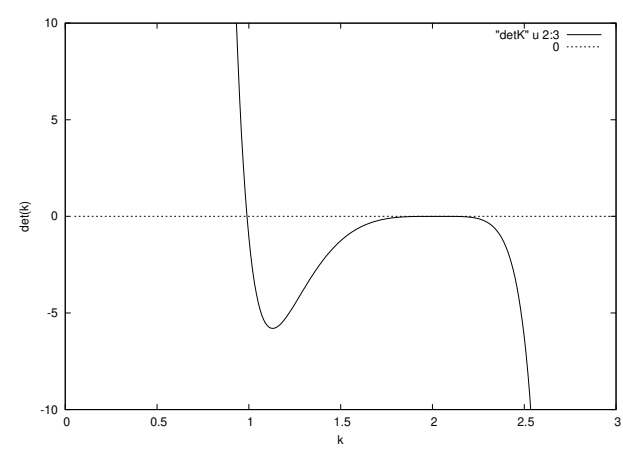

Figure 2: The determinant for the star-like quantum graph.

In the numerical experiments to determine the zeros of det $h(k)$, there is a tendency that a particular point of $k$ attracts the sampling particles, but other zeros are forsaken, if the initial configuration of the particles is not well-chosen. It is undesirable to leave some zeros of $\operatorname{det}(k)$ neglected by the sampling. The tactics to circumvent this undesirable situation is simple: we can give the limit to the span of the coordinates of the sampling particles, and we do the sampling in small pieces of the limited zones. As for the choice of the objective function, we could add the step-function-like one to the original function $(\operatorname{det}(k))$, which might be defined by

$$
\theta(k)= \begin{cases}1 & k<k_{l} \\ 0 & k_{l} \leq k \leq k_{u} \\ 1 & k_{u}<k\end{cases}
$$

In the particle swarm simulation, we use the initial conditions given in Table 1 . In the simulations given in the forthcoming sections, we employ the same setting.

\begin{tabular}{|c|c|}
\hline Number of particles & 10 \\
$\mathrm{~W}$ & 1.4 \\
$\mathrm{C} 1$ & 0.7 \\
$\mathrm{C} 2$ & 0.7 \\
$\chi$ & 1 \\
Initial coordinates & $0 \leq X_{i} \leq 0.01$ \\
Initial velocities & $\left|v_{i}\right| \leq 0.000001$ \\
\hline
\end{tabular}

Table 1: The initial parameters of the simulation. As the coordinates and velocities, we set the initial random values of each directions in the range defined in the table. 
The typical behaviour of the swarm simulation is shown in Table.2. We see that the convergence to the optimum is sooth.

\begin{tabular}{ccccl} 
Iter & Average & Variation & Group Best & Objective function \\
\hline 1 & 0.004615 & 0.000012 & 0.009733 & 17341147919996737941162426368.000000 \\
11 & 0.091911 & 0.000025 & 0.098030 & 15423759002567858.000000 \\
21 & 0.343053 & 0.000191 & 0.370078 & 1173915429.229820 \\
31 & 0.865516 & 0.000703 & 0.897965 & 434.552037 \\
41 & 0.990161 & 0.000620 & 0.990017 & 0.000321 \\
51 & 0.987437 & 0.000043 & 0.989916 & 0.000041 \\
61 & 0.989118 & 0.000016 & 0.989854 & 0.000000 \\
71 & 0.989574 & 0.000001 & 0.989862 & 0.000000 \\
81 & 0.989844 & 0.000000 & 0.989862 & 0.000000 \\
91 & 0.989844 & 0.000000 & 0.989859 & 0.000000 \\
101 & 0.989888 & 0.000000 & 0.989859 & 0.000000 \\
111 & 0.989854 & 0.000000 & 0.989859 & 0.000000 \\
121 & 0.989857 & 0.000000 & 0.989859 & 0.000000 \\
131 & 0.989860 & 0.000000 & 0.989859 & 0.000000 \\
141 & 0.989859 & 0.000000 & 0.989859 & 0.000000 \\
151 & 0.989859 & 0.000000 & 0.989859 & 0.000000
\end{tabular}

Table 2: The behavior of the particle swarm optimization. The columns (from left to right) show the following properties for each iteration: the average of the coordinates of the particles; their variations; the coordinates of the current best particle position; the objective function of the current best particle.

\subsection{Optimization on nodes}

Let us try more complicated problems. We use the same star-like graph used in the previous section. The target is to minimize the ratio of the amplitude of the wavefunction on the nodes, defined by

$$
\frac{\left|\phi_{1}\right|}{\left|\phi_{0}\right|}
$$

with the constraint det $h(k)=0$. We execute the optimization with respect to the potential $\lambda_{1}$ at node 1. In this model, if det $h(k) \neq 0$, the solution of Eq. (7) is the trivial one. Hence the wavefunction is not obtained and the objective function is not well-defined. To circumvent this trouble, we modify the problem slightly in the following way. First, instead of Eq. (7), we consider the eigenvalue problem

$$
\sum_{j} h_{j i} x_{j}=e \cdot x_{i} .
$$


Second, We select a $\phi_{i}$ from the solutions of the above eigenvalue problem such that it should be the eigenvevtor corresponding to the eigenvalue closest to zero. The objective function is then defined by

$$
f\left(k, \lambda_{1}\right)=|\operatorname{det} h(k)|^{2}+\frac{\left|\phi_{1}\right|}{\left|\phi_{0}\right|} .
$$

At the minimum of this objective function, we anticipate the minimum of $\frac{\left|\phi_{1}\right|}{\left|\phi_{0}\right|}$ and the fulfillment of $\operatorname{det} h(k)=0$.

The statistical feature of the particle swarms is given in Table 3 . We see that the computation is successful.

\begin{tabular}{c|cccccc} 
Iter & Aver1 & Aver2 & Group Best1 & Group Best2 & Objective function & det \\
\hline 1 & 0.004987 & 0.004447 & 0.009139 & 0.001820 & $6.81 \mathrm{E}+27$ & $6.81 \mathrm{E}+27$ \\
21 & 0.517388 & -0.250155 & 0.554074 & -0.267241 & $1.28+\mathrm{E} 06$ & $1.28 \mathrm{E}+06$ \\
41 & 0.942385 & -0.449224 & 0.942918 & -0.449484 & 0.692088 & 0.0003 \\
61 & 0.943959 & -0.449968 & 0.943076 & -0.449557 & 0.691785 & 0.0000 \\
81 & 0.943113 & -0.449570 & 0.943084 & -0.449557 & 0.691784 & 0.00000 \\
101 & 0.943059 & -0.449303 & 0.943049 & -0.449270 & 0.691766 & 0.0000 \\
121 & 0.942875 & -0.447623 & 0.942866 & -0.447535 & 0.691666 & 0.0000 \\
141 & 0.942108 & -0.441724 & 0.942079 & -0.441507 & 0.691269 & 0.0000
\end{tabular}

Table 3: The behavior of the particle swarm optimization. The columns (from left to right) show the following properties for each iteration: the averages of the two coordinates of the particles ( $k$ and $\lambda_{1}$ ); the coordinates of the current best particle position; the objective function; the corresponding determinant. COnerning the coordinates of the sampling particles, the convergence of the variations below $1.0 E-06$ is affirmed in the last stages of the simulation.

The obtained best coordinates is indeed a minimum as is shown in Fig. 3. 


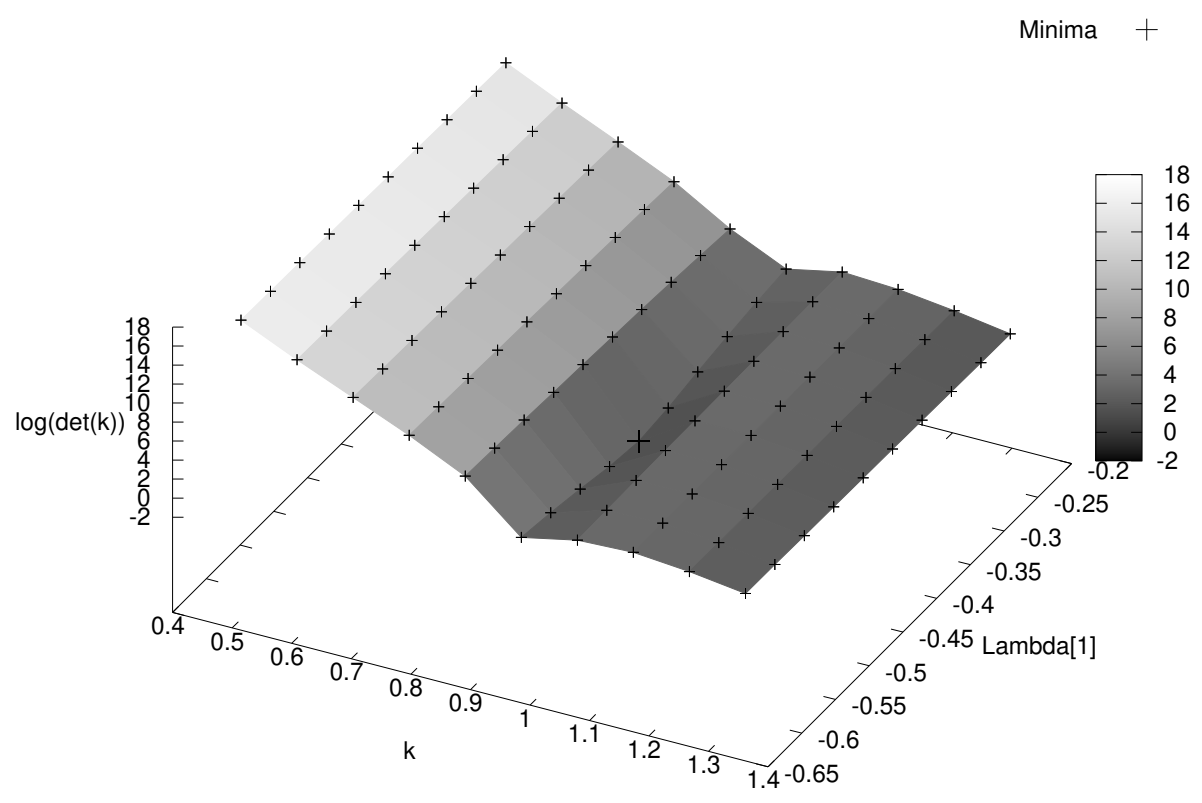

Figure 3: The feature of the objective function in the neighborhood of the minimum. The minimum is indicated by a cross symbol.

\subsection{Optimization on edges}

We show a slightly different example of the optimization. Let us define the square norm of the wavefunction locally on the $i$ th edge

$$
N(i):=\int_{0}^{L_{b}}\left|\psi_{b}(x ; k)\right|^{2} d x .
$$

We try to minimize the following:

$$
f=\frac{N(1)}{\sum_{i=1}^{B} N(i)},
$$

with the constraint

$$
\operatorname{det} h(k)=0 \text {, }
$$

with respect to $\lambda_{1}$ (the paramter defined at the 1 st node). The objective function is defined in the same manner as in the previous section.

As is shown in Table. 4, the optimization works well, having achieved the zero of the determinant. 


\begin{tabular}{c|cccccc} 
Iter & Aver1 & Aver2 & Group Best1 & Group Best2 & Objective function & det \\
\hline 1 & 0.004987 & 0.004447 & 0.009139 & 0.001820 & $6.81 \mathrm{E}+27$ & $6.81 \mathrm{E}+27$ \\
21 & 0.517388 & -0.250155 & 0.554074 & -0.267241 & $1.28 \mathrm{E}+06$ & $1.28 \mathrm{E}+06$ \\
41 & 0.942385 & -0.449224 & 0.942918 & -0.449484 & 0.267996 & 0.00025 \\
61 & 0.943969 & -0.449973 & 0.943070 & -0.449551 & 0.267723 & 0.00000 \\
81 & 0.943096 & -0.449564 & 0.943074 & -0.449553 & 0.267723 & 0.00000 \\
101 & 0.943079 & -0.449556 & 0.943074 & -0.449554 & 0.267723 & 0.00000 \\
121 & 0.943075 & -0.449560 & 0.943075 & -0.449561 & 0.267721 & 0.00000 \\
141 & 0.943100 & -0.449688 & 0.943101 & -0.449693 & 0.267701 & 0.00000
\end{tabular}

Table 4: The behaviour of the particle swarm optimization. The columns (from left to right) show the following properties for each iteration : the average of the two coordinates of the particles ( $k$ and lambda); the coordinates of the current best particle position; the objective function; the corresponding determinant. The convergence of the variations below $1.0 E-06$ is affirmed in the last stages of the simulation.

\section{A discrete problem}

In this section, we investigate the optimization of a discrete model of quantum graphs. In a graph, we can connect and disconnect the edges freely. Then we can manage the property of quantum graphs by the addition or the removal of the components. As a test problem, we adopt the star-like graph again in the previous sections, and we use the freedom of the edge-connection as the controllable variables in the optimization. As for the objective function, we minimize the lowest energy spectrum (or, equivalently, the wave-number $k$ ). We depict the graph through the following variables:

- If the nodes $\mathrm{i}$ and $\mathrm{j}$ are connected, $\mathrm{c}[\mathrm{i}, \mathrm{j}]=1$;

- Else $c[i, j]=0$.

We adopt the genetic algorithm and we choose those variables as the genes. In the implementation, we determine the energy spectrum by a simple way as follows: we divide a finite range of wavenumber $\mathrm{k}$ into finite numbers of uniform meshes, and we compute the determinant in each of them. By checking the change of the signs of the determinant, we determine the place of zero approximately, within the admissible accuracy. (We use the meshes of the width 0.01 ; and if $h(x) h(y)<0$ for two sample points at both ends of a mesh, we guess the existence of the zero around $(x+y) / 2$, and use this point in the evaluation of the objective function.) In the test problem, we set an additional condition: the lengths $l_{i}$ of the edges 
are randomly chosen in the range of $0.5<l<1.5$, and they are fixed in all trials of the optimization. Using those conditions, we try to determine the optimized graph, for which the wave-number $k$ corresponding to the lowest energy should be as close as possible to the target values. In the genetic algorithm, we choose the standard measures: the elite selection; the crossover with the priority to the genes of better fitness; the mutation. The result is given in Table 5. The GA works well for discrete optimization. The result tallies with the physical interpretation: on account of the repeated removal of the edges, the electron is confined in narrower spaces and the consequence is the rise of the eigenenergy.

\begin{tabular}{c|c|c}
$K$-Target & The best gene & $K$-Obtained \\
\hline 0.1 & $(1,1,1,1,1)$ & 0.47 \\
0.2 & $(1,1,1,1,1)$ & 0.47 \\
0.3 & $(1,1,1,1,1)$ & 0.47 \\
0.4 & $(1,1,1,1,1)$ & 0.47 \\
0.5 & $(1,1,1,1,1)$ & 0.47 \\
0.6 & $(0,1,1,1,1)$ & 0.60 \\
0.7 & $(1,1,1,1,0)$ & 0.69 \\
0.8 & $(0,1,1,1,0)$ & 0.81 \\
0.9 & $(1,1,0,1,0)$ & 0.88 \\
1.0 & $(0,1,0,1,0)$ & 1.03 \\
1.1 & $(1,0,1,0,0)$ & 1.10 \\
1.2 & $(0,0,1,0,0)$ & 1.19 \\
1.3 & $(0,1,0,0,0)$ & 1.32 \\
1.4 & $(1,0,0,0,0)$ & 1.36 \\
1.5 & $(0,0,0,0,0)$ & 1.46
\end{tabular}

Table 5: The result of the quantum optimization by GA. Each row shows the target value of $K$, the obtained best GENE, and the actually obtained $K$ of the lowest eigenvalue from the best gene. The integers in the genes indicate $(c[0,1], \ldots c[0,5])$, namely, the connection between the center and the five peripheral nodes in the star-shaped graph.

\section{Conclusion}

In this article, we demonstrated the efficacy of the heuristic algorithm applied to the optimization of quantum graphs. We have adopted the particle swarm optimization and genetic algorithm; we have pointed out the particular feature to be treated carefully in the computation of the quantum graphs; we have ascertained that the adopted method has worked well. The merit of our approach is as follows: 
in the heuristic method, it is not necessary to compute derivatives of the objective functions, and we can anticipate the application to various problems. To conclude this article, we should note that we have only investigated the efficacy of the genetic algorithm and the particle swarm optimization independently, but no more. We would suppose problems to find the quantum graph structures with the optimized edge-connections and the optimized continuous parameters. This sort of problem should be solved by the collaboration of genetic algorithm and particle swarm optimization: the former shall optimize the discrete problem and the latter shall adjust the parameters in the continuous space. This type of generalization shall be the target of future study.

\section{Acknowledgment}

The sample programs used in the numerical experiments are now available in public from GitHub (www.github.com/kikuchiichio/OptQuantumGraph). Though we (the authors) disclaim the copyright and leave the programs in the public domain, free to distributions and modifications, we shall be grateful to you if you refer to this article in case you make scientific publications using them.

\section{References}

[1] Gregory Berkolaiko and Peter Kuchment. Introduction to quantum graphs. American Mathematical Soc., 2013.

[2] Sven Gnutzmann and Uzy Smilansky. Quantum graphs: Applications to quantum chaos and universal spectral statistics. Advances in Physics, 55(5-6):527625, 2006.

[3] Trevor Hastie, Robert Tibshirani, and Martin Wainwright. Statistical learning with sparsity: the lasso and generalizations. CRC press, 2015. 